\title{
Consolidating the Effects of Waking and Sleep on Motor-Sequence Learning
}

\author{
Timothy P. Brawn, ${ }^{1}$ Kimberly M. Fenn, ${ }^{2}$ Howard C. Nusbaum, ${ }^{1}$ and Daniel Margoliash ${ }^{1,3}$ \\ ${ }^{1}$ Department of Psychology, University of Chicago, Chicago, Illinois 60637, ${ }^{2}$ Department of Psychology, Michigan State University, East Lansing, Michigan \\ 48824, and ${ }^{3}$ Department of Organismal Biology and Anatomy, University of Chicago, Chicago, Illinois 60637
}

\begin{abstract}
Sleep is widely believed to play a critical role in memory consolidation. Sleep-dependent consolidation has been studied extensively in humans using an explicit motor-sequence learning paradigm. In this task, performance has been reported to remain stable across wakefulness and improve significantly after sleep, making motor-sequence learning the definitive example of sleep-dependent enhancement. Recent work, however, has shown that enhancement disappears when the task is modified to reduce task-related inhibition that develops over a training session, thus questioning whether sleep actively consolidates motor learning. Here we use the same motorsequence task to demonstrate sleep-dependent consolidation for motor-sequence learning and explain the discrepancies in results across studies. We show that when training begins in the morning, motor-sequence performance deteriorates across wakefulness and recovers after sleep, whereas performance remains stable across both sleep and subsequent waking with evening training. This pattern of results challenges an influential model of memory consolidation defined by a time-dependent stabilization phase and a sleep-dependent enhancement phase. Moreover, the present results support a new account of the behavioral effects of waking and sleep on explicit motor-sequence learning that is consistent across a wide range of tasks. These observations indicate that current theories of memory consolidation that have been formulated to explain sleep-dependent performance enhancements are insufficient to explain the range of behavioral changes associated with sleep.
\end{abstract}

\section{Introduction}

The acquisition of a new skill initiates a process of memory formation wherein the newly formed memory trace is consolidated into a more stable and strengthened form. The consolidation of memories is widely believed to benefit from sleep (see Walker, 2005; Diekelmann and Born, 2010 for reviews). Though evidence from multiple domains has supported a role for sleep in memory processing, sleep-dependent consolidation has been studied most extensively using an explicit motor-sequence learning paradigm. In this task, participants repeatedly type a short sequence (e.g., 4-1-3-2-4), and the number of correctly typed sequences improves significantly during training. $\mathrm{Nu}$ merous studies have reported that while task performance remains stable across a $12 \mathrm{~h}$ waking retention period, significant performance enhancements are observed after comparable retention intervals that include sleep (e.g., Walker et al., 2002, 2003; Korman et al., 2003; Fischer et al., 2005; Hotermans et al., 2006; Korman et al., 2007).

The interpretation of these experiments, however, has recently been challenged by observations indicating that the reported postsleep performance enhancements are an artifact of the study design (Rickard et al., 2008; Cai and Rickard, 2009). The emergence of performance fatigue and reactive inhibition, which

Received June 25, 2010; revised Aug. 27, 2010; accepted Aug. 27, 2010.

This work was supported in part by National Institute of Mental Health Grant MH059831 and National Institute on Deafness and Other Communication Disorders Grant DC007206.

Correspondence should be addressed to Timothy P. Brawn, University of Chicago, 1027 East 57th Street, Chicago, IL 60637. E-mail: tbrawn@uchicago.edu.

DOI:10.1523/JNEUROSCI.3295-10.2010

Copyright $\odot 2010$ the authors $\quad 0270-6474 / 10 / 3013977-06 \$ 15.00 / 0$ is expressed as a worsening of performance within each $30 \mathrm{~s}$ trial, were argued to impair performance during the training and posttraining test trials (Rickard et al., 2008). The training procedure appeared to play a critical role in producing the appearance of sleep-dependent enhancement because the sleep-enhancement effect was eliminated when the experimental design was modified to reduce task-dependent confounds. These results were interpreted as indicating that sleep does not enhance motor performance and have been used to question the existence of an active memory consolidation process unique to sleep (Rickard et al., 2008).

The effects of waking and sleep retention on motor-sequence consolidation nonetheless remain unresolved. Though Rickard et al. (2008) provided evidence that sleep does not enhance motor-sequence learning, performance in the modified experiment was not tested after waking retention. Thus, it is unclear whether sleep had any effect on motor-sequence performance because the skill level before sleep was unknown. In other learning experiments (albeit using different perceptual or sensorimotor tasks) in humans and starlings, performance degraded across a waking retention interval and then recovered after sleep (Fenn et al., 2003; Brawn et al., 2008, 2010). Here we trained and tested participants on the same motor-sequence learning task using both the original (massed training) and modified (spaced training) experimental procedures. Additionally, participants were tested after a 5 min rest period following the posttraining test (cf. Hotermans et al., 2006) to further explore inhibition effects. The results presented here provide a new, coherent account of the behavioral effects of waking and sleep on explicit motorsequence learning, ultimately challenging existing models of sleep-dependent consolidation. 
Table 1. Experimental design

\begin{tabular}{|c|c|c|c|c|c|}
\hline Condition & Training & Retention 1 & Posttest 1 & Retention 2 & Posttest 2 \\
\hline AM-massed $(n=15)$ & 8:30 -9:30 A.M. & $12 \mathrm{~h}$ wake & 9:00 -9:30 P.M. & $12 \mathrm{~h}$ sleep & 9:00-9:30 A.M. \\
\hline PM-massed $(n=14)$ & 8:30 -9:30 P.M. & 12 h sleep & 9:00-9:30 A.M. & $12 \mathrm{~h}$ wake & 9:00-9:30 P.M. \\
\hline AM-spaced $(n=20)$ & 8:30 -9:30 A.M. & $12 \mathrm{~h}$ wake & 9:00 -9:30 P.M. & $12 \mathrm{~h}$ sleep & 9:00 -9:30 A.M. \\
\hline PM-spaced $(n=14)$ & 8:30 -9:30 P.M. & $12 \mathrm{~h}$ sleep & 9:00 -9:30 A.M. & $12 \mathrm{~h}$ wake & 9:00-9:30 P.M. \\
\hline
\end{tabular}

\section{Materials and Methods}

\section{Participants}

Right-handed, nonmusician, University of Chicago students $(n=85,56$ female) aged 18 to 30 (mean age $=20.5$ ) provided written informed consent and were financially compensated for participation. To maximize the accuracy of self-reporting, participants were not instructed on how to behave while outside the lab. The data from 22 participants were not analyzed: one did not complete the experiment, one dataset was erased due to a computer error, two were left-handed, two consumed alcohol before training, and 16 took naps during the waking retention interval.

\section{Motor sequence task}

The sequential finger-tapping task entailed using the left (nondominant) hand to type a five-element sequence (4-1-3-2-4) on a computer keyboard as quickly and accurately as possible for the duration of each trial. The numeric sequence was displayed on the screen during every trial. Each key press produced an " " on the screen to indicate the key press had been recorded without providing accuracy feedback. The experimental task was written in Matlab using the Psychophysics Toolbox (Brainard, 1997).

\section{Experimental design}

Participants were assigned to one of four experimental conditions (Table 1). Each condition included a training session and two posttest sessions that occurred 12 and $24 \mathrm{~h}$ after training. Morning sessions began between 8:30 and 9:30 A.M.; evening sessions began between 8:30 and 9:30 P.M. Half of the participants were trained in the morning and half were trained in the evening. Two groups (one morning and one evening) received massed training, wherein each trial lasted $30 \mathrm{~s}$ with $30 \mathrm{~s}$ of rest between trials. The other two groups (one morning and one evening) received spaced training, wherein each trial lasted $10 \mathrm{~s}$ with $30 \mathrm{~s}$ of rest between trials. The number of trials was different for the massed and spaced conditions (Table 2), but the total time spent typing the sequences was identical for each condition.

\section{Performance measures}

Completed sequences and error rate. Each correct five-element sequence was extracted from the series of key presses within a trial to produce a "sequence completed" score. Key presses not part of a correct sequence were counted as errors, and the "error rate" score was calculated as the ratio of errors to total key presses. Key presses that were part of a correct, but incomplete, sequence at the end of a trial (e.g., 4-1-3) were included in the total key-press count but not as errors or sequences completed. The pretest consisted of the first $30 \mathrm{~s}$ trial for the massed conditions or the average of the first three $10 \mathrm{~s}$ trials for the spaced conditions. The remaining tests (posttraining, postrest, postretention test 1 , and postretention test 2) consisted of the average of two $30 \mathrm{~s}$ trials for the massed conditions or the average of six $10 \mathrm{~s}$ trials for the spaced conditions.

Response times. The timing of every key press was recorded, and the average response time was calculated over $10 \mathrm{~s}$ intervals for each trial. For the massed conditions, three response times were computed for every trial corresponding to the first, second, and third $10 \mathrm{~s}$ segment of the $30 \mathrm{~s}$ trial. For the spaced conditions, each response time corresponded to a single $10 \mathrm{~s}$ trial. To explore changes in response times over a single trial in the massed conditions, a response time difference score was computed by subtracting the response time of the first $10 \mathrm{~s}$ segment from the third $10 \mathrm{~s}$ segment. A similar score was computed for the spaced conditions by subtracting the corresponding $10 \mathrm{~s}$ segments (e.g., subtracting the response time of trial 1 from trial 3).

\section{Statistical analysis}

Two-way repeated-measures ANOVA with time-of-training (A.M. or P.M.) and time (pretest, posttrain, postrest, posttest1, and posttest2)
Table 2. Session procedure

\begin{tabular}{|c|c|c|}
\hline Trial type & Number of trials & Trial duration \\
\hline \multicolumn{3}{|c|}{ Massed-training procedure } \\
\hline \multicolumn{3}{|c|}{ Training session } \\
\hline Warm-up & 1 & $10 s$ \\
\hline Pretest & 1 & $30 \mathrm{~s}$ \\
\hline Training & 9 & $30 \mathrm{~s}$ \\
\hline Posttrain test & 2 & $30 s$ \\
\hline Rest period & 1 & $5 \mathrm{~min}$ \\
\hline Warm-up & 1 & $10 \mathrm{~s}$ \\
\hline Postrest test & 2 & $30 s$ \\
\hline \multicolumn{3}{|l|}{ Posttest session 1} \\
\hline Warm-up & 1 & $10 s$ \\
\hline Posttest 1 & 2 & $30 s$ \\
\hline \multicolumn{3}{|l|}{ Posttest session 2} \\
\hline Warm-up & 1 & $10 s$ \\
\hline Posttest 2 & 2 & $30 s$ \\
\hline \multicolumn{3}{|c|}{ Spaced-training procedure } \\
\hline \multicolumn{3}{|c|}{ Training session } \\
\hline Warm-up & 1 & $10 s$ \\
\hline Pretest & 3 & $10 s$ \\
\hline Training & 27 & $10 s$ \\
\hline Posttrain test & 6 & $10 s$ \\
\hline Rest period & 1 & $5 \mathrm{~min}$ \\
\hline Warm-up & 1 & $10 \mathrm{~s}$ \\
\hline Postrest test & 6 & $10 s$ \\
\hline \multicolumn{3}{|l|}{ Posttest session 1} \\
\hline Warm-up & 1 & $10 \mathrm{~s}$ \\
\hline Posttest 1 & 6 & $10 s$ \\
\hline \multicolumn{3}{|l|}{ Posttest session 2} \\
\hline Warm-up & 1 & $10 s$ \\
\hline Posttest 2 & 6 & $10 s$ \\
\hline
\end{tabular}

factors were applied separately to the massed-training conditions and to the spaced-training conditions to assess performance changes for number of sequences completed and error rate. Bonferroni-corrected posttests were used to evaluate differences between specific tests. Paired $t$ tests were used to detect changes in response time difference scores from the posttraining test to the postrest test. Unpaired $t$ tests were used to compare changes in response time difference scores between the massed and spaced conditions and to compare Stanford Sleepiness Scores for participants who completed the experimental sessions in the morning or evening. One-way ANOVA was used to check for differences in sleep duration. All statistical analyses were computed using GraphPad Prism 5 (GraphPad Software).

\section{Sleep data}

Participants were allowed keep their normal sleep schedule and selfrecorded their sleep patterns for $5 \mathrm{~d}$ before the experiment. The amount of sleep on the night of the study ranged from $6.8 \pm 1.3 \mathrm{~h}$ (mean $\pm \mathrm{SD}$ ) to $7.6 \pm 1.1 \mathrm{~h}$ across the conditions, and there were no significant differences in sleep duration. Participants completed the Stanford Sleepiness Scale at each session, and there were no significant differences.

\section{Results}

Performance progression of massed and spaced conditions To investigate the effects of waking and sleep retention on motorsequence performance following learning, participants were trained and tested on an explicit motor-sequence finger-tapping 
A

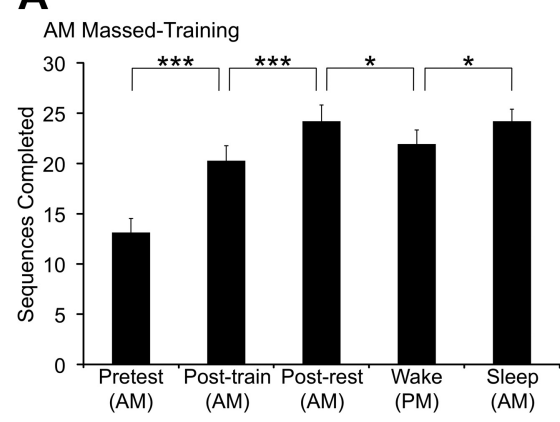

C

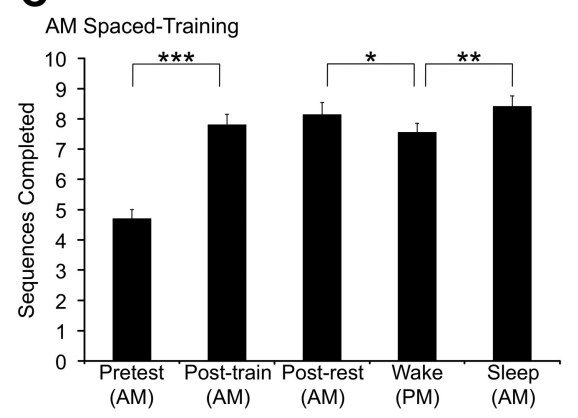

B

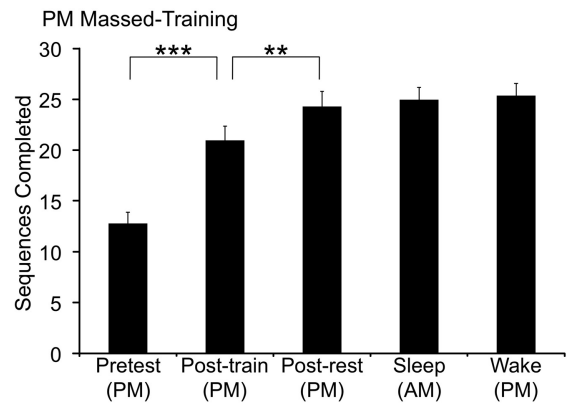

D

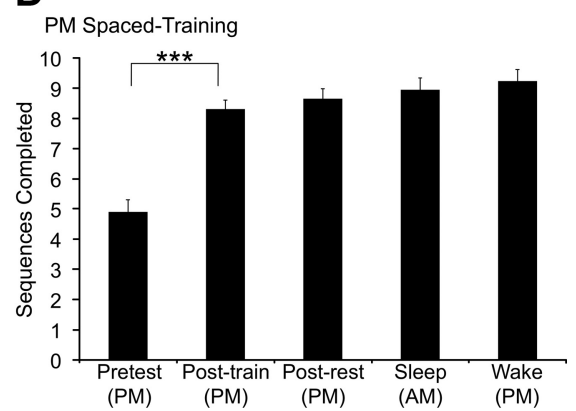

Figure 1. Motor-sequence performance across test trials. Performance was measured as the number of correctly completed sequences during the test trials. The completed-sequence scores for the spaced conditions $(\boldsymbol{C}, \boldsymbol{D})$ are approximately one-third of the massed conditions $(\boldsymbol{A}, \boldsymbol{B})$ because the spaced-condition scores were averaged over $10 \mathrm{~s}$ trials rather than $30 \mathrm{~s}$ trials. $\boldsymbol{A}, \mathrm{A} . \mathrm{M}$. massed-training condition. B, P.M. massed-training condition. C, A.M. spaced-training condition. D, P.M. spaced-training condition. Data are the means $\pm \operatorname{SEM}\left({ }^{*} p<0.05 ;{ }^{* *} p<0.01 ;{ }^{* * *} p<0.001\right)$.

task. Participants were assigned to one of four conditions and performance was measured before training (pretest) and then at four posttest time points: at the end of training, after a 5 min rest period, and at 12 and $24 \mathrm{~h}$ after training (Table 1). Both training types (massed and spaced) produced significant differences for number of sequences completed across the pretest and subsequent posttests (massed, $F_{(4,108)}=101.30$; spaced, $F_{(4,128)}=$ 144.40; $p<0.0001$ for both). Neither training type displayed an effect for time of training (massed, $F_{(1,108)}=0.29, p=0.59$; spaced, $\left.F_{(1,128)}=2.33, p=0.13\right)$. The spaced-training conditions showed a time by time-of-training interaction $\left(F_{(4,128)}=2.80\right.$, $p<0.05)$, though the massed-training conditions did not $\left(F_{(4,108)}=\right.$ $1.80, p=0.13)$. There were no significant error rate changes in any condition ( $p>0.16$ for all).

The massed-training conditions entailed training and testing trials that lasted $30 \mathrm{~s}$ with $30 \mathrm{~s}$ of rest between each trial (cf. Walker et al., 2002). In the A.M.-massed condition (Fig. $1 A$ ), the number of sequences completed for each $30 \mathrm{~s}$ trial increased by $7.1 \pm 0.9$ (mean \pm SEM) from the pretest to the posttraining test, representing a significant improvement after training $\left(t_{(108)}=\right.$ 7.48; $p<0.001)$. After a 5 min rest period, performance further increased by a significant $3.9 \pm 0.7$ sequences $\left(t_{(108)}=4.13 ; p<\right.$ 0.001). Performance subsequently decreased following a $12 \mathrm{~h}$ waking retention interval by a significant $2.3 \pm 0.9$ sequences $\left(t_{(108)}=2.38 ; p<0.05\right)$ and then significantly improved by $2.3 \pm$ 0.6 sequences after a $12 \mathrm{~h}$ retention interval that included a night of sleep $\left(t_{(108)}=2.38 ; p<0.05\right)$. For the P.M.-massed condition (Fig. $1 B)$, participants displayed a significant improvement of $8.2 \pm 1.1$ sequences after training $\left(t_{(108)}=8.29 ; p<0.001\right)$. The 5 min rest period produced an additional significant increase of $3.3 \pm 0.8$ sequences $\left(t_{(108)}=3.37 ; p<0.01\right)$. Performance remained stable thereafter, increasing by only $0.7 \pm 0.4$ sequences after a night of sleep $\left(t_{(108)}=0.69 ; p=\right.$ $0.49)$ and by $0.4 \pm 0.7$ sequences after a full day awake $\left(t_{(108)}=0.40 ; p=0.69\right)$.

The spaced-training conditions entailed training and testing trials that lasted $10 \mathrm{~s}$ with $30 \mathrm{~s}$ of rest between each trial (cf. Rickard et al., 2008). In the A.M.-spaced condition (Fig. 1C), the number of sequences completed in each $10 \mathrm{~s}$ trial increased by $3.1 \pm 0.3$ after training, representing a significant performance improvement $\left(t_{(128)}=12.64 ; p<0.001\right)$. After a $5 \mathrm{~min}$ rest period, performance showed a nonsignificant increase of $0.3 \pm$ 0.3 sequences $\left(t_{(128)}=1.36 ; p=0.17\right)$. Performance subsequently decreased by a significant $0.6 \pm 0.3$ sequences over a $12 \mathrm{~h}$ waking retention interval $\left(t_{(128)}=2.38\right.$; $p<0.05)$ and then significantly improved by $0.9 \pm 0.2$ sequences following sleep $\left(t_{(128)}=3.49 ; p<0.01\right)$. For the P.M.spaced condition (Fig. 1D), participants displayed a significant improvement of $3.4 \pm 0.3$ sequences after training $\left(t_{(128)}=\right.$ $11.61 ; p<0.001)$. The $5 \mathrm{~min}$ rest period produced a nonsignificant increase of $0.3 \pm 0.1$ sequences $\left(t_{(128)}=1.17 ; p=\right.$ 0.24). Performance remained stable thereafter, exhibiting a nonsignificant increase of $0.3 \pm 0.2$ sequences after sleep $\left(t_{(128)}=1.01 ; p=0.31\right)$ and of $0.3 \pm 0.2$ sequences after a full day awake $\left(t_{(128)}=0.98 ; p=0.33\right)$.

\section{Reactive inhibition and the postrest performance enhancement}

The A.M.- and P.M.-massed conditions displayed significant performance enhancements after a 5 min rest period following the posttraining test, whereas neither spaced condition exhibited an enhancement following the rest period. An analysis of the key-press response times clarifies why the massed conditions showed a postrest performance enhancement and the spaced conditions did not. Inspection of response times for the A.M.massed condition (Fig. $2 \mathrm{~A}$ ) shows a clear pattern beginning with the second training trial wherein response times get progressively slower (i.e., reactive inhibition) during each $10 \mathrm{~s}$ segment of the $30 \mathrm{~s}$ trials. By the posttraining test, the response time difference (see Materials and Methods) between the first and third $10 \mathrm{~s}$ segments of the test trials was $51.8 \pm 9.1 \mathrm{~ms}$. This pattern of reactive inhibition was dramatically attenuated after the 5 min rest period, where the response time difference for the postrest test was $23.5 \pm 7.6 \mathrm{~ms}$. The P.M.-massed condition exhibited a similar pattern (Fig. $2 \mathrm{~B}$ ), where the response time difference was reduced from $35.4 \pm 14.9 \mathrm{~ms}$ for the posttraining test to $19.8 \pm$ $6.1 \mathrm{~ms}$ for the postrest test. Together, the response time difference for the massed conditions was reduced from $43.4 \pm 8.3 \mathrm{~ms}$ on the posttraining trials to $21.6 \pm 4.7 \mathrm{~ms}$ on the postrest trials. This indicates that each key press at the end of the postrest trials was on average $\sim 22 \mathrm{~ms}$ faster than key presses at the end of the posttraining trials, demonstrating a significant response time improvement after the rest period $\left(t_{(28)}=2.43 ; p<0.05\right)$.

In contrast, inspection of the A.M.-spaced condition (Fig. 2 A) shows a smooth progression of response times across training and testing with no evidence of reactive inhibition. The response 


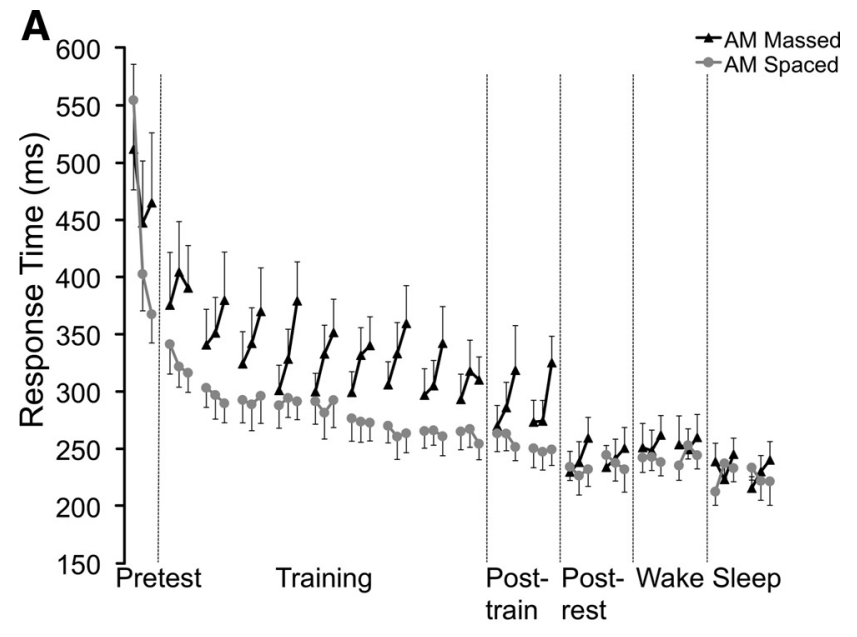

B

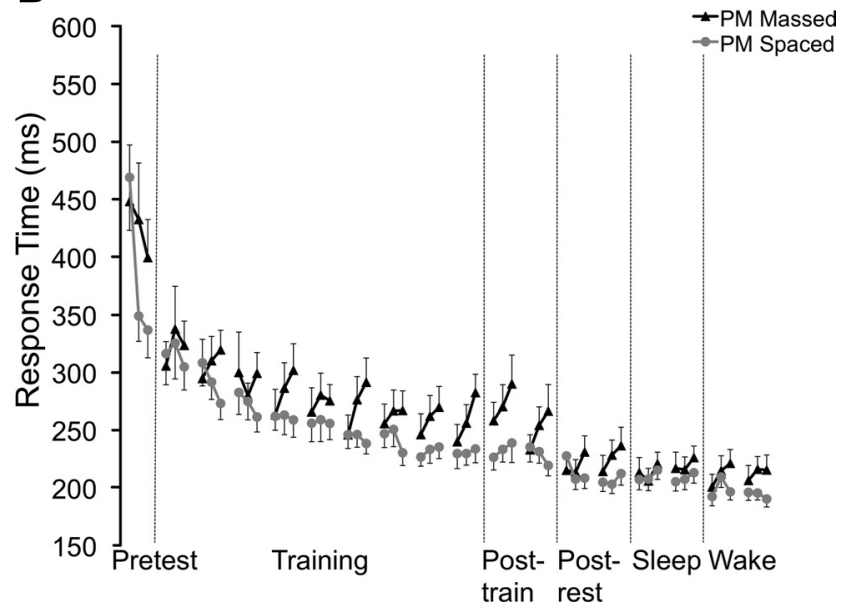

Figure 2. Response times across trials. Each data point represents the mean response time for each key press over a 10 s interval. For the spaced conditions, each data point corresponds to the mean response time for each $10 \mathrm{~s}$ trial. For the massed conditions, each $30 \mathrm{~s}$ trial was separated into three $10 \mathrm{~s}$ blocks. The data points are combined into triplets, where the massed condition triplets correspond to the $30 \mathrm{~s}$ trials and the spaced triplets are matched for consistency but represent independent trials. $\boldsymbol{A}$, Response times for A.M.-massed and -spaced conditions. $\boldsymbol{B}$, Response times for P.M.-massed and -spaced conditions.

time difference in the A.M.-spaced condition was $-4.4 \pm 6.2 \mathrm{~ms}$ for the corresponding $10 \mathrm{~s}$ segments on the posttraining trials and was $-9.6 \pm 5.4 \mathrm{~ms}$ for the corresponding postrest trials. Likewise, the response time difference for the P.M.-spaced condition (Fig. $2 B$ ) was $-4.7 \pm 3.5 \mathrm{~ms}$ for the corresponding $10 \mathrm{~s}$ segments on the posttraining trials and was $-3.1 \pm 4.7 \mathrm{~ms}$ for the corresponding postrest trials. Together, the spaced conditions exhibited a change of $2.4 \pm 4.9 \mathrm{~ms}$. This demonstrates that each key press at the end of the postrest trials was only $\sim 2$ ms faster than key presses at the end of the posttraining trials. Thus, the 5 min rest period did not produce a response time performance benefit $\left(t_{(33)}=0.49 ; p=0.62\right)$. The improvement in response time difference scores was significantly greater for the massed conditions than for the spaced conditions $\left(t_{(61)}=1.98 ; p=0.05\right)$.

\section{Lack of circadian effects}

Performance changes across the multiple test sessions could potentially be explained by natural variation in motor performance at different times of day rather than as the result of time spent awake or asleep. However, there was no difference in the pretest performance between the A.M.- and P.M.-massed conditions $\left(t_{(27)}=0.19 ; p=0.85\right)$ or the A.M.- and P.M.-spaced conditions $\left(t_{(32)}=0.49 ; p=0.68\right)$, indicating that time of day had no effect on initial performance level. Moreover, there was no difference in the amount of learning during the training session for the A.M.and P.M.-massed $\left(t_{(27)}=0.72 ; p=0.48\right)$ or A.M.- and P.M.spaced $\left(t_{(32)}=0.65 ; p=0.52\right)$ conditions, indicating that time of training had no effect on the ability to learn motor sequences. Accordingly, circadian factors on motor performance do not explain the present results.

\section{Discussion}

\section{Patterns of explicit motor-sequence consolidation}

We have demonstrated a pattern of memory consolidation that challenges a substantial body of prior research on the effects of waking and sleep on explicit motor-sequence learning. We found that performance deteriorated significantly across the day and then recovered after a night of sleep when participants were trained in the morning. In contrast, performance remained stable across both a night of sleep and subsequent waking when training occurred in the evening. Therefore, sleep restored motorsequence performance after it had deteriorated during a period of wakefulness before sleep, and sleep stabilized the motor memory against degradation during a subsequent day of wakefulness. Importantly, sleep did not enhance motor-sequence learning beyond the performance level achieved after training. These results differ from the extensively reported pattern of consolidation in which motor-sequence learning is said to remain unchanged across wakefulness but is enhanced after a night of sleep (e.g., Walker et al., 2002, 2003; Korman et al., 2003; Fischer et al., 2005; Hotermans et al., 2006; Korman et al., 2007).

The difference between the current findings and previous research stems from our inclusion of a test session 5 min after the end of training. Hotermans et al. (2006) reported that performance on this task was enhanced when participants were retested after a 5 or $30 \mathrm{~min}$ rest period following the posttraining test. This postrest enhancement was replicated in our A.M.- and P.M.massed conditions but not in the A.M.- or P.M.-spaced conditions. An analysis of the key-press response times showed substantial reactive inhibition in the massed conditions, similar to that found by Rickard et al. (2008), which was significantly attenuated after the 5 min rest period and coincided with a significant performance enhancement. In contrast, the spaced conditions, which completed shorter trials and received more rest during the training session, did not show evidence of reactive inhibition during training and, consequently, did not exhibit a postrest enhancement. The cause of reactive inhibition in the massed conditions is uncertain, as it could result from the accumulation of fatigue, interference, or attentional factors. Nonetheless, it is clear that reactive inhibition profoundly hinders motor-sequence performance on the posttraining test, an effect that can be greatly reduced with spaced training or a brief rest period before the posttraining test.

Accordingly, the postrest test is a more accurate indicator of motor-sequence skill acquired during training than the posttraining test because the confounding effects of reactive inhibition are substantially reduced. We conclude that previous studies significantly underestimated motor-sequence performance at the end of training by relying on the posttraining test as a marker of motor-sequence skill, resulting in the illusory pattern of stable performance across wakefulness and enhancement after sleep. Indeed, if the postrest test is ignored, the results from the A.M.and P.M.-massed conditions replicate the previously reported pattern of wake-state stabilization and sleep-state enhancement. 
Ultimately, the patterns of consolidation demonstrated here suggest that an influential model of memory consolidation (Walker, 2005), which asserts that procedural memories experience a time-dependent stabilization phase and a sleep-dependent enhancement phase, cannot adequately explain the performance changes found after wakefulness and sleep in explicit motorsequence learning.

\section{Implications for existing models of sleep-dependent consolidation}

The pattern of wake-state deterioration followed by sleep-state recovery and stabilization is consistent with other sleep consolidation studies, as the same result has been found for perceptual learning of synthetic speech (Fenn et al., 2003) and sensorimotor learning (Brawn et al., 2008). Moreover, although sleep has been commonly reported to enhance visual texture discrimination learning (e.g., Gais et al., 2000), it is plausible that texture discrimination studies may suffer from task-structure confounds that result in similar fatigue or reactive inhibition and could potentially follow the pattern of consolidation demonstrated here. Indeed, texture discrimination training and testing sessions entail $>1000$ trials, and performance has been shown to deteriorate if participants are retested multiple times during the day, implicating fatigue in the visual system as a critical factor in the reported pattern of performance changes (Mednick et al., 2002). Additionally, similar inhibition effects were recently discovered for motor pursuit learning (Rieth et al., 2010), suggesting that confounding inhibition effects may be common in procedural tasks. Collectively, these studies further challenge the procedural memory consolidation model defined by a time-dependent stabilization and a sleep-dependent enhancement phase.

While our results confirm that sleep does not enhance motorsequence learning (cf. Rickard et al., 2008; Cai and Rickard, 2009), they also suggest active processes during sleep. During the first $12 \mathrm{~h}$ retention period, performance in the A.M.training conditions deteriorated across wakefulness and performance in the P.M.-training conditions remained unchanged across sleep, which is consistent with a passive process of reduced interference during sleep (Wixted, 2004; Rickard et al., 2008). However, there was also significant performance recovery after sleep in the A.M.-training conditions. Similar to other procedural tasks (Fenn et al., 2003; Brawn et al., 2008), sleep restored performance lost over waking retention. Perhaps access to memories acquired early in the day was blocked by subsequent daytime activity (i.e., daytime formation of additional memories), with access improving during sleep when no additional memories were formed. This could be viewed as a complex form of reduced interference. Alternatively, perhaps some memories were lost during waking retention but the remaining memories formed a trace sufficiently robust to create new "memories" during sleep, and the new "memories" helped to restore performance. These are distinctions that are amenable to experimental disambiguation, but in either case, they represent active sleep processes. Finally, sleep following training prevented performance loss during subsequent waking retention. This process of consolidation may be distinct from the process of performance restoration, but it cannot be explained simply by a lack of interference; rather, it suggests an active mechanism of sleep-dependent stabilization (Korman et al., 2007).

Existing theories of memory consolidation do not fully account for the pattern of consolidation described here. The synaptic homeostasis hypothesis (Tononi and Cirelli, 2006) only partially explains the experimental results. The performance deterioration over waking retention in the A.M.-training conditions could result from a decrease in signal-to-noise ratio due to synaptic potentiation during the day. Synaptic downscaling during sleep could then increase the signal-to-noise ratio, producing postsleep performance recovery. Yet, synaptic downscaling should also increase the signal-to-noise ratio and produce postsleep performance improvements in the P.M.-training conditions, and this did not occur. Likewise, neural reactivation, a process whereby patterns of neural activity that are expressed during waking behaviors are replayed during subsequent sleep, is commonly thought to underlie sleep-dependent consolidation (Diekelmann and Born, 2010). Reactivation could act as an offline period of rehearsal, enabling the synaptic strengthening of newly formed memory traces. However, this would not explain why the A.M.-training conditions exhibited a significant performance change across sleep but the P.M.-training conditions did not. If sleep-dependent consolidation is achieved through reactivation-induced synaptic strengthening (i.e., synaptic consolidation), performance after sleep should be significantly better than performance during the previous evening, regardless of when learning occurred during the day. The present results, however, could be compatible with active systems consolidation theory, which argues that reactivation is involved in transferring new memory traces from temporary to long-term storage during sleep (Diekelmann and Born, 2010). Though systems consolidation, via reactivation-induced memory transfer, has generally been applied to hippocampus-dependent memory, it could be relevant for nondeclarative tasks like motor-sequence learning, which has been shown to undergo systems-level changes following sleep (Fischer et al., 2005), and is potentially consistent with a pattern of sleep-dependent recovery and stabilization.

Overall, the present results demonstrate that explicit motor-sequence learning, which has been the paradigmatic example of sleep-dependent enhancement, is not enhanced by sleep but rather follows a pattern of deterioration over waking retention before sleep and recovery and stabilization of performance as a result of sleep. This pattern of consolidation challenges the claims of a sleep-enhancement effect and indicates the need for modification of existing models of sleepdependent consolidation.

\section{References}

Brainard DH (1997) The psychophysics toolbox. Spat Vis 10:433-436.

Brawn TP, Fenn KM, Nusbaum HC, Margoliash D (2008) Consolidation of sensorimotor learning during sleep. Learn Mem 15:815-819.

Brawn TP, Nusbaum HC, Margoliash D (2010) Sleep-dependent consolidation of auditory discrimination learning in adult starlings. J Neurosci 30:609-613.

Cai DJ, Rickard TC (2009) Reconsidering the role of sleep for motor memory. Behav Neurosci 123:1153-1157.

Diekelmann S, Born J (2010) The memory function of sleep. Nat Rev Neurosci 11:114-126.

Fenn KM, Nusbaum HC, Margoliash D (2003) Consolidation during sleep of perceptual learning of spoken language. Nature 425:614-616.

Fischer S, Nitschke MF, Melchert UH, Erdmann C, Born J (2005) Motor memory consolidation in sleep shapes more effective neuronal representations. J Neurosci 25:11248-11255.

Gais S, Plihal W, Wagner U, Born J (2000) Early sleep triggers memory for early visual discrimination skills. Nat Neurosci 3:1335-1339.

Hotermans C, Peigneux P, Maertens de Noordhout A, Moonen G, Maquet P (2006) Early boost and slow consolidation in motor skill learning. Learn Mem 13:580-583.

Korman M, Raz N, Flash T, Karni A (2003) Multiple shifts in the representation of a motor sequence during the acquisition of skilled performance. Proc Natl Acad Sci U S A 100:12492-12497. 
Korman M, Doyon J, Doljansky J, Carrier J, Dagan Y, Karni A (2007) Daytime sleep condenses the time course of motor memory consolidation. Nat Neurosci 10:1206-1213.

Mednick SC, Nakayama K, Cantero JL, Atienza M, Levin AA, Pathak N, Stickgold R (2002) The restorative effect of naps on perceptual deterioration. Nat Neurosci 5:677-681.

Rickard TC, Cai DJ, Rieth CA, Jones J, Ard MC (2008) Sleep does not enhance motor sequence learning. J Exp Psychol Learn Mem Cogn 34:834-842.

Rieth CA, Cai DJ, McDevitt EA, Mednick SC (2010) The role of sleep and practice in implicit and explicit motor learning. Behav Brain Res $214: 470-474$.
Tononi G, Cirelli C (2006) Sleep and synaptic homeostasis. Sleep Med Rev $10: 49-62$.

Walker MP (2005) A refined model of sleep and the time course of memory formation. Behav Brain Sci 28:51-64.

Walker MP, Brakefield T, Morgan A, Hobson JA, Stickgold R (2002) Practice with sleep makes perfect: sleep-dependent motor skill learning. Neuron 35:205-211.

Walker MP, Brakefield T, Seidman J, Morgan A, Hobson JA, Stickgold R (2003) Sleep and the time course of motor skill learning. Learn Mem 10:275-284.

Wixted JT (2004) The psychology and neuroscience of forgetting. Annu Rev Psychol 55:235-269. 